\title{
AN INTRINSIC DESCRIPTION OF SHAPE
}

\author{
JOSE M. R. SANJURJO
}

\begin{abstract}
We give in this paper a description of the shape category of compacta in terms of multivalued maps. We introduce the notion of a multi-net and prove that the shape category of compacta is isomorphic to the category $H N$ whose objects are metric compacta and whose morphisms are homotopy classes of multi-nets. This description is intrinsic in the sense that it does not make use of external elements such as ANR-expansions or embeddings in appropriate AR-spaces. We present many applications of this new formulation of shape.
\end{abstract}

\section{INTRODUCTION}

Shape theory was introduced in 1968 by $\mathrm{K}$. Borsuk [3] as a new classification of compact metric spaces which takes into account their global properties and neglects the local ones. Borsuk's main notion was that of a fundamental sequence which plays the same role as the notion of mapping in homotopy theory. Fundamental sequences are defined in terms of the neighborhoods of compacta embedded in a convenient AR-space. Shape theory was later developed for more general spaces. Of special importance is the Mardešić-Segal approach [16] where shape theory is based on inverse systems of ANRs. In this approach, shapes are defined for arbitrary Hausdorff compacta. Maps between such systems are defined as well as a notion of homotopy of such maps. This homotopy relation classifies maps between ANR-systems and these classes are called shape maps. In both cases the description of shape involves some external elements like ambient spaces where to embed the compacta or ANR-expansions.

The aim of this paper is to present a new description of the shape category of compacta in which no use is made of such external elements. Our description is intrinsic in the sense that we only use maps (or sequences of maps) between compacta as opposed to the usual descriptions where maps are defined between some objects associated to the compacta. Our maps are multivalued. We introduce the notion of a multi-net between compacta which consists of a sequence of upper-semicontinuous multivalued maps progressively smaller, becoming more and more similar to a single-valued map. This notion is related to

Received by the editors January 19, 1989 and, in revised form, November 17, 1989.

1980 Mathematics Subject Classification (1985 Revision). Primary 54C56, 54C60; Secondary $55 \mathrm{P} 55$.

Key words and phrases. Small multivalued map, multi-net, simple multi-net, approximative map, refinable map, strong shape equivalence, internal movability.

Partially supported by CAICYT. The results of this paper were obtained while the author was visiting the Department of Mathematics of the University of Liverpool. The author wants to express his gratitude to the members of the Department for their hospitality. 
that of a fine multivalued map that we introduced in [21]. The main result in [21], where we described shape morphisms as homotopy classes of fine multivalued maps, is used in this paper in the proof of Theorem 2 which is an essential step towards the description of the shape category of compacta in terms of multivalued maps that we present in Theorem 3. As a corollary we obtain a simple characterization of the maps inducing shape equivalences and use this result to give a new proof of Kato's Theorem about refinable maps whose codomain is a FANR [10]. We also give a characterization of the inclusions inducing strong shape equivalences. In the last section of the paper we study in detail the homotopy classes of multi-nets between internally movable compacta and prove that they admit a nice kind of representatives that we call simple multi-nets. Hence, shape morphisms between internally movable compacta can be represented by these simple multi-nets. In order to prove this result we must first give a new characterization of internally movable compacta in terms of multivalued maps.

We should mention here that another description of the shape category of compacta has recently been given by the author [20] in terms of $\varepsilon$-continuity, extending some ideas of Felt [9] and answering a question raised by Klee [11]. In [13 and 14] it is shown that shape morphisms can also be interpreted as the path components of a certain topological space of approximative maps.

All the spaces considered in the paper are metric and we always use the letter $d$ to represent the corresponding distance. By a multivalued function $F: X \rightarrow$ $Y$ we mean a function assigning to each point $x \in X$ a nonempty closed subset $F(x)$ of $Y . F$ is said to be upper semicontinuous if for each point $x \in X$ and for each neighborhood $V$ of $F(x)$ in $Y$ there exists a neighborhood $U$ of $x$ in $X$ such that $F(U)=\bigcup\left\{F\left(x^{\prime}\right) \mid x^{\prime} \in U\right\}$ is contained in $V$. In the sequel we shall refer to upper semicontinuous multivalued functions as multivalued maps. A multivalued map $F: X \rightarrow Y$ is said to be $\varepsilon$-small provided that diameter $(F(x))<\varepsilon$ for all $x \in X$. We shall use the letter $\delta$ to represent the diameter of a set. Two $\varepsilon$-small multivalued maps $F, G: X \rightarrow Y$ are $\varepsilon$ multihomotopic if there exists an $\varepsilon$-small multivalued map $H: X \times I \rightarrow Y$ such that $H(x, 0)=F(x)$ and $H(x, 1)=G(x)$ for every $x \in X$. The multivalued map $H$ is then called an $\varepsilon$-multihomotopy connecting $F$ and $G$. This notion applies even in the case when $F$ and $G$ are single-valued. Obviously, $\varepsilon$-multihomotopy is an equivalence relation.

We shall often assume, without loss of generality, that compact metric spaces are subsets of the Hilbert cube, $Q$. If $X_{0}$ is a closed subset of a compactum $X$, by $B\left(X_{0}, \varepsilon\right)$ we mean the generalized ball (in $X$ ) of radius $\varepsilon$. If $F, G: X \rightarrow Y$ are multivalued maps between compacta, the notation $d(F, G)<\varepsilon$ means that distance $(F(x), G(x))<\varepsilon$ for every $x \in X$. The reader is referred to the texts of Borsuk [2], Dydak-Segal [7] and Mardešić-Segal [17] for information about shape theory.

\section{MUlTi-NETS AND SHAPE MORPHISMS}

To state our results, we need first to introduce some of the basic definitions.

Definition 1. Let $X$ and $Y$ be compact metric spaces. A multi-net from $X$ to $Y$ is a sequence of multivalued maps $F_{n}: X \rightarrow Y$ such that for every $\varepsilon>0$ there is an index $n_{0}$ such that $F_{n}$ is $\varepsilon$-multihomotopic to $F_{n+1}$ for every $n \geq n_{0}$. 
A multi-net will be represented by the notation $\mathbf{F}=\left(F_{n}\right): X \rightarrow Y$.

In the following definition we recall the notion of a fine multivalued map that we introduced in [21].

Definition 2. A fine multivalued map from $X$ to $Y$ is a multivalued map $\alpha: X \times \mathbf{R}_{+} \rightarrow Y$ such that for every $\varepsilon>0$ there is a $t_{0} \in \mathbf{R}_{+}=[0, \infty)$ such that $\alpha_{\mid X \times\left[t_{0}, \infty\right)}$ is $\varepsilon$-small.

Shape morphisms between compacta $X$ and $Y$ lying in the Hilbert cube, $Q$, can be described as homotopy classes of approximative maps from $X$ towards $Y$. The notion of an approximative map was introduced by Borsuk in [3]. An approximative map $\mathbf{f}=\left\{f_{k}, X \rightarrow Y\right\}$ is a sequence of continuous functions $f_{k}: X \rightarrow Q$ such that for every neighborhood $V$ of $Y$ in $Q$ there is an index $k_{0}$ such that $f_{k}$ is homotopic to $f_{k+1}$ for every $k \geq k_{0}$. We say that an approximative map $\mathbf{f}=\left\{f_{k}, X \rightarrow Y\right\}$ is obtained from a multi-net $\mathbf{F}=\left(F_{n}\right)$ : $X \rightarrow Y$ by an infinitesimal translation if for every $\varepsilon>0$ there is an index $n_{0}$ such that $d\left(f_{n}, F_{n}\right)<\varepsilon$ for every $n \geq n_{0}$.

Theorem 1. For every multi-net $\mathbf{F}=\left(F_{n}\right): X \rightarrow Y$ there is an approximative map $\mathbf{f}=\left\{f_{k}, X \rightarrow Y\right\}$ that is obtained from $\mathbf{F}$ by an infinitesimal translation. Moreover, every two approximative maps obtained from $\mathbf{F}$ by an infinitesimal translation are homotopic.

We omit the proof of Theorem 1 since it is an easy consequence of the following Approximation Lemma which was proved (in a slightly different form) in [21]. For the sake of completeness we shall give here a proof of this new form of the lemma.

Approximation Lemma. For every $\varepsilon$-small multivalued map $F$ from a compactum $Y$ to $Q$ there is a (single-valued) map $f: Y \rightarrow Q$ such that $d(f, F)<$ $\varepsilon$.

Proof. We can assume that $Y$ consists of more than one point. Since $F$ is upper-semicontinuous there exists for every $y \in Y$ a neighborhood $V$ of $y$ in $Y$ such that $F(V) \subset B(F(y),(\varepsilon-\delta(F(y))) / 3)$. Hence, there is a finite open covering $Y_{1}, \ldots, Y_{n}$ of $Y$ such that $Y_{i} \neq Y, Y_{i} \neq \phi$, and $\delta\left(F\left(Y_{i}\right)\right)<\varepsilon$ for $i=1, \ldots, n$. Define for every $i$ a function $\lambda_{i}: Y \rightarrow \mathbf{R}$ by the expression $\lambda_{i}(y)=d\left(y, Y-Y_{i}\right) / \sum_{j=1}^{n} d\left(y, Y-Y_{j}\right)$. Clearly $\lambda_{i}$ is continuous for every $i$ and $\lambda_{i}(y) \neq 0$ if and only if $y \in Y_{i}$. We choose for every $i$ a point $x_{i} \in F\left(Y_{i}\right)$ and define a function $f: Y \rightarrow Q$ by the expression $f(y)=\sum_{i=1}^{n} \lambda_{i}(y) x_{i}$. The function $f$ is obviously continuous. Moreover, if $y \in Y$ suppose that $Y_{1}, \ldots, Y_{k}$ are the only members of the covering containing $y$. Then we have $f(y)=\lambda_{1}(y) x_{1}+\cdots+\lambda_{k}(y) x_{k}$ and $\lambda_{1}(y)+\cdots+\lambda_{k}(y)=1$. If $x \in F(y)$ we also have that $x=\lambda_{1}(y) x+\cdots+\lambda_{k}(y) x$ and, as a consequence, $d(x, f(y)) \leq$ $\lambda_{1}(y)\left\|x-x_{1}\right\|+\cdots+\lambda_{k}(y)\left\|x-x_{k}\right\|<\varepsilon$, here we use the norm of the Hilbert space in which $Q$ is supposed to be embedded. This shows that $d(f, F)<\varepsilon$ and the proof of the lemma is complete.

We state now some corollaries which are a consequence of the Approximation Lemma. We only give the proof of the first of them since the rest can be obtained in quite a similar way.

Corollary 1. For every compactum $Y$ and for every $\varepsilon>0$ there is a $\eta>0$ such 
that if $F, G: X \rightarrow Y$ are $\eta$-close (i.e. $d(F, G)<\eta$ ) and $\eta$-small multivalued maps then $F$ and $G$ are $\varepsilon$-multihomotopic.

Corollary 2. If $Y$ is a compact ANR then for every $\varepsilon>0$ there is a $\eta>0$ such that for every $\eta$-small multivalued map $F: X \rightarrow Y$ there is a single-valued map $f: X \rightarrow Y$ with $d(f, F)<\varepsilon$.

Corollary 3. If $Y \in A N R$ then there is an $\varepsilon>0$ such that every two singlevalued maps $f, g: X \rightarrow Y$ which are $\varepsilon$-multihomotopic are homotopic.

Corollary 4. If $Y \in F A N R$ then there is an $\varepsilon>0$ such that every two singlevalued maps $f, g: X \rightarrow Y$ which are $\varepsilon$-close are $\eta$-multihomotopic for every $\eta>0$.

Proof of Corollary 1. Let $\varepsilon>0$ be given and let $V$ be an ANR-neighborhood of $Y$ in $Q$ such that $V \subset B(Y, \varepsilon / 3)$. Pick up a positive number $\varepsilon^{\prime}<\varepsilon$ such that every two $\varepsilon^{\prime}$-close maps to $V$ are homotopic and $B\left(Y, \varepsilon^{\prime}\right) \subset V$. Take $\eta=\varepsilon^{\prime} / 6$ and suppose that $F, G: X \rightarrow Y$ are $\eta$-small and $\eta$-close multivalued maps. By the Approximation Lemma there are maps $f, g: X \rightarrow Q$ such that $d(f, F)<\eta$ and $d(g, G)<\eta$. It is easy to see that this implies that $f$ and $g$ take values in $V$ and $f$ and $g$ are $\varepsilon^{\prime}$-close and, hence, homotopic. Suppose $H: X \times I \rightarrow V$ is a homotopy connecting $f$ and $g$. We define the multivalued map $\widetilde{H}: X \times I \rightarrow Y$ by the expression $\widetilde{H}(x, t)=\{y \in Y \mid$ $d(y, H(x, t)) \leq \varepsilon / 3\}$; obviously $\widetilde{H}$ is $\varepsilon$-small. Moreover, $F(x) \subset \widetilde{H}(x, 0)$ and $G(x) \subset \widetilde{H}(x, 1)$ for every $x \in X$ and this clearly implies that $F$ and $G$ are $\varepsilon$-multihomotopic. This completes the proof of the corollary.

If $\mathbf{F}=\left(F_{n}\right): X \rightarrow Y$ and $\mathbf{G}=\left(G_{n}\right): X \rightarrow Y$ are multi-nets we say that $\mathbf{F}$ and $\mathbf{G}$ are homotopic provided that for every $\varepsilon>0, \mathbf{F}_{n}$ is $\varepsilon$-multihomotopic to $G_{n}$ for almost every $n$. Similarly two fine multivalued maps $\alpha, \beta: X \times \mathbf{R}_{+} \rightarrow$ $Y$ are homotopic provided that for every $\varepsilon>0$ there is a $t_{0} \in \mathbf{R}_{+}$such that $\alpha_{\mid X \times\left[t_{0}, \infty\right]}$ and $\beta_{\mid X \times\left[t_{0}, \infty\right]}$ are $\varepsilon$-multihomotopic. The relation of homotopy of multi-nets and fine multivalued maps is an equivalence relation.

If $\alpha: X \times \mathbf{R}_{+} \rightarrow Y$ is a fine multivalued map we define a multi-net $\phi(\alpha)=$ $\mathbf{F}=\left(F_{n}\right): X \rightarrow Y$ by the expression $F_{n}=\alpha_{\mid X \times\{n\}}$. We can also consider a function assigning to each multi-net $\mathbf{F}=\left(F_{n}\right): X \rightarrow Y$ an approximative map $w(\mathbf{F})=\mathbf{f}=\left\{f_{k}, X \rightarrow Y\right\}$ which is an infinitesimal translation of $\mathbf{F}$. According to Theorem 1, the homotopy class of $w(\mathbf{F})$ does not depend on the particular function $w$.

Now we shall state our next result. We denote by $C_{f}(X, Y)$ the set of all homotopy classes of fine multivalued maps from $X$ to $Y$ and by $C_{n}(X, Y)$ the set of all homotopy classes of multi-nets from $X$ to $Y . \operatorname{By} \operatorname{Sh}(X, Y)$ we mean the set of all shape morphisms from $X$ to $Y$. We use the symbol [ ] to denote homotopy classes. In the proof of the theorem we use the main result of [21], according to which there is a natural one-to-one correspondence between $C_{f}(X, Y)$ and $\operatorname{Sh}(X, Y)$.

Theorem 2. (a) The correspondence $[\alpha] \rightarrow[\phi(\alpha)]$ defines a bijection $\phi_{X, Y}$ : $C_{f}(X, Y) \rightarrow C_{n}(X, Y)$. (b) The correspondence $[\mathbf{F}] \rightarrow[w(\mathbf{F})]$ defines a bijection $\Omega_{X, Y}: C_{n}(X, Y) \rightarrow \operatorname{Sh}(X, Y)$.

Proof. We shall prove that $\phi_{X, Y}$ and $\Omega_{X, Y}$ are well-defined functions and that $\phi_{X, Y}$ is bijective. Then we get that $\Omega_{X, Y}$ is also bijective from the fact, proved 
in [21], that the correspondence $[\alpha] \rightarrow[w \phi(\alpha)]$ defines a bijection between $C_{f}(X, Y)$ and $\operatorname{Sh}(X, Y)$.

The function $\phi_{X, Y}$ is clearly well-defined since homotopic fine multivalued maps define by restriction homotopic multi-nets. To see that $\Omega_{X, Y}$ is welldefined, suppose that the multi-nets $\mathbf{F}$ and $\mathbf{F}^{\prime}$ are homotopic and $\mathbf{f}=w(\mathbf{F})$, $\mathbf{f}^{\prime}=w\left(\mathbf{F}^{\prime}\right)$. Let $V$ be an ANR-neighborhood of $Y$ in $Q$ and consider an $\varepsilon>0$ and a $k_{0}$ such that

(1) $d\left(f_{k_{0}}, F_{k_{0}}\right)<\varepsilon / 3$ and $d\left(f_{k_{0}}^{\prime}, F_{k_{0}}^{\prime}\right)<\varepsilon / 3$,

(2) $f_{k} \simeq f_{k_{0}}$ and $f_{k}^{\prime} \simeq f_{k_{0}}^{\prime}$ in $V$ for $k \geq k_{0}$,

(3) there is an $\varepsilon / 3$-multihomotopy, $H$, connecting $F_{k_{0}}$ and $F_{k_{0}}^{\prime}$,

(4) every two $\varepsilon$-close maps taking values in $V$ are homotopic, and

(5) $B(Y, \varepsilon) \subset V$.

Now consider a (single-valued) map $h: X \times[0,1] \rightarrow Q$ such that

$$
d(h(x, t), H(x, t))<\varepsilon / 3
$$

for every $(x, t) \in X \times I$. Clearly $h(X \times[0,1]) \subset V$. Moreover $d\left(f_{k_{0}}, h_{0}\right)<\varepsilon$ and $d\left(f_{k_{0}}^{\prime}, h_{1}\right)<\varepsilon$. Hence $f_{k_{0}} \simeq h_{0} \simeq h_{1} \simeq f_{k_{0}}^{\prime}$ in $V$ and, as a consequence, $[\mathbf{f}]=\left[\mathbf{f}^{\prime}\right]$ and $\Omega_{X, Y}$ is well-defined.

The injectivity of $\phi_{X, Y}$ is a consequence of the fact that if $\alpha_{\mid X \times\left[t_{0}, \infty\right]}$ is $\varepsilon$-small, then the expression

$$
H(x, t, s)=\alpha\left(x, s t_{0}+(1-s) t\right)
$$

defines an $\varepsilon$-multihomotopy between $\alpha_{\mid X \times\left[t_{0}, \infty\right)}$ and the multivalued function $\alpha^{\prime}: X \times\left[t_{0}, \infty\right) \rightarrow Y$ defined by $\alpha^{\prime}(x, t)=\alpha\left(x, t_{0}\right)$. Finally $\phi_{X, Y}$ is surjective since for every multi-net $\mathbf{F}=\left(F_{n}\right): X \rightarrow Y$ there is a fine multivalued map $\alpha: X \times \mathbf{R}_{+} \rightarrow Y$ such that $\alpha_{\mid X \times\{n\}}=F_{n}$. The restriction $\alpha_{\mid X \times[n, n+1]}$ is defined by means of the $\varepsilon$-multihomotopy connecting $F_{n}$ and $F_{n+1}$.

\section{THE HOMOTOPIC CATEGORY OF COMPACTA AND MULTINETS}

We shall show in this section that if we consider the class of all compact metric spaces and the homotopy classes of multi-nets between them, we are able to define a notion of composition in such a way that we get a category isomorphic to the shape category of compacta. First we remark that if $\mathbf{F}=\left(F_{n}\right): X \rightarrow Y$ and $\mathbf{G}=\left(G_{n}\right): Y \rightarrow Z$ are multi-nets we cannot define its composition in the straightforward way $\mathbf{G F}=\left(G_{n} F_{n}\right): X \rightarrow Z$ because very simple examples show that this sequence of multivalued maps is not, in general, a multi-net. We can however introduce a notion of composition of the homotopy classes [F] and [G] as follows: Consider a null sequence of positive numbers $\varepsilon_{1} \geq \varepsilon_{2} \geq$ $\cdots \geq \varepsilon_{n} \cdots$ such that $G_{n}$ is $\varepsilon_{n_{0}}$-multihomotopic to $G_{n_{0}}$ for every $n \geq n_{0}$. Select another null sequence $\mu_{1} \geq \mu_{2} \geq \cdots \geq \mu_{n} \geq \cdots$ such that $\delta\left(G_{n}(K)\right)<\varepsilon_{n}$ whenever $K$ is a set in $Y$ with $\delta(K)<\mu_{n}$. Next consider a sequence of indices $k_{1}<\cdots<k_{n} \cdots$ such that $F_{k}$ is $\mu_{n}$-multihomotopic to $F_{k_{n}}$ for every $k \geq k_{n}$. We define $[\mathbf{G}] \circ[\mathbf{F}]=\left[\left(G_{n} F_{k_{n}}\right)\right]$. In our next result we prove that in this way we get a category.

Theorem 3. If we consider the class of all metric compacta and the homotopy classes of multi-nets between them with the notion of composition previously introduced, we get a category $H N$ which is isomorphic to the shape category of compacta. 
Proof. We show first that $\left(G_{n} F_{k_{n}}\right): X \rightarrow Z$ is a multi-net. Let $\varepsilon>0$ be given and consider an index $n_{0}$ such that $\varepsilon_{n_{0}}<\varepsilon$. Then, if $n \geq n_{0}$ there is an $\varepsilon_{n_{0}}$-multihomotopy $H_{n}: Y \times I \rightarrow Z$ such that $H_{n}(y, 0)=G_{n_{0}}(y)$, $H_{n}(y, 1)=G_{n}(y)$ for every $y \in Y$. Consider a $\mu>0$ such that $\delta\left(H_{n}(K)\right)<$ $\varepsilon_{n_{0}}$ whenever $K$ is a subset of $Y \times I$ with $\delta(K)<\mu$ and select an $m \geq$ $n$ such that $\mu_{m}<\mu$. Then it is easy to see that $G_{n_{0}} F_{k_{m}}$ and $G_{n} F_{k_{m}}$ are $\varepsilon_{n_{0}}$-multihomotopic. Since $F_{k_{m}}$ and $F_{k_{n}}$ are $\mu_{n}$-multihomotopic, we have that $G_{n} F_{k_{m}}$ and $G_{n} F_{k_{n}}$ are $\varepsilon_{n}$-multihomotopic and, analogously, $G_{n_{0}} F_{k_{m}}$ and $G_{n_{0}} F_{k_{n_{0}}}$ are $\varepsilon_{n_{0}}$-multihomotopic. Hence, we have that $G_{n} F_{k_{n}}$ and $G_{n_{0}} F_{k_{n_{0}}}$ are $\varepsilon$-multihomotopic for every $n \geq n_{0}$ and, as a consequence, $\left(G_{n} F_{k_{n}}\right)$ is a multinet.

Now we show that the homotopy class of $\left(G_{n} F_{k_{n}}\right)$ does not depend on the choices made in the composition or on the representatives of the homotopy classes $[\mathbf{F}]$ and $[\mathbf{G}]$. Suppose that the multi-nets $\mathbf{F}^{\prime}=\left(F_{n}^{\prime}\right): X \rightarrow Y$ and $\mathbf{G}^{\prime}=\left(G_{n}^{\prime}\right): Y \rightarrow Z$ are homotopic to $\mathbf{F}$ and $\mathbf{G}$ respectively. Consider a null sequence of positive numbers $\varepsilon_{1}^{\prime} \geq \cdots \geq \varepsilon_{n}^{\prime} \geq \cdots$ such that $G_{n}^{\prime}$ is $\varepsilon_{n_{0}}^{\prime}$ multihomotopic to $G_{n_{0}}^{\prime}$ for every $n \geq n_{0}$. Select another sequence $\mu_{1}^{\prime} \geq \cdots \geq$ $\mu_{n}^{\prime} \geq \cdots$ such that $\delta\left(G_{n}^{\prime}(K)\right)<\varepsilon_{n}^{\prime}$ whenever $K$ is a set in $Y$ with $\delta(K)<\mu_{n}^{\prime}$ and consider a sequence of indices $k_{1}^{\prime}<\cdots<k_{n}^{\prime}<\cdots$ such that $F_{k}^{\prime}$ is $\mu_{n}^{\prime}$ multihomotopic to $F_{k_{n}^{\prime}}^{\prime}$ for every $k \geq k_{n}^{\prime}$. We shall prove that the multi-net $\left(G_{n}^{\prime} F_{k_{n}^{\prime}}^{\prime}\right)$ is homotopic to $\left(G_{n} F_{k_{n}}\right)$. Let $\varepsilon>0$ be given and consider an index $n_{0}$ such that $\max \left\{\varepsilon_{n_{0}}, \varepsilon_{n_{0}}^{\prime}\right\}<\varepsilon$ and $G_{n_{0}}$ is $\varepsilon$-multihomotopic to $G_{n_{0}}^{\prime}$. An argument similar to that used in the first part of the proof shows that $G_{n} F_{k_{n}}$ is $\varepsilon$-multihomotopic to $G_{n_{0}} F_{k_{n_{0}}}$ and $G_{n}^{\prime} F_{k_{n}^{\prime}}^{\prime}$, is $\varepsilon$-multihomotopic to $G_{n_{0}}^{\prime} F_{k_{n_{0}}^{\prime}}^{\prime \prime}$ for every $n \geq n_{0}$. Hence we only need to prove that $G_{n_{0}} F_{k_{n_{0}}}$ and $G_{n_{0}}^{\prime} F_{k_{n_{0}}}^{\prime \prime}$ are $\varepsilon$-multihomotopic. Since $G_{n_{0}}$ and $G_{n_{0}}^{\prime}$ are $\varepsilon$-multihomotopic it can be seen as before that there is an index $m \geq \max \left\{k_{n_{0}}, k_{n_{0}}^{\prime}\right\}$ such that $G_{n_{0}} F_{m}$ and $G_{n_{0}}^{\prime} F_{m}$ are $\varepsilon$-multihomotopic. Using the fact that $\mathbf{F}$ and $\mathbf{F}^{\prime}$ are homotopic we can also assume that $m$ is large enough so that $G_{n_{0}}^{\prime} F_{m}$ and $G_{n_{0}}^{\prime} F_{m}^{\prime}$ are $\varepsilon$-multihomotopic. Moreover, the fact that $F_{m}$ is $\mu_{n_{0}}$-multihomotopic to $F_{k_{n_{0}}}$ implies that $G_{n_{0}} F_{m}$ and $G_{n_{0}} F_{k_{n_{0}}}$ are $\varepsilon_{n_{0}}$-multihomotopic. Similarly $G_{n_{0}}^{\prime} F_{m}^{\prime}$ and $G_{n_{0}}^{\prime} F_{k_{n_{0}}^{\prime}}^{\prime}$ are $\varepsilon_{n_{0}}^{\prime}$-multihomotopic. This implies that $G_{n_{0}} F_{k_{n_{0}}}$ and $G_{n_{0}}^{\prime} F_{k_{n_{0}}}^{\prime}$ are $\varepsilon$ multihomotopic and, as a consequence, the multi-nets $\left(G_{n} F_{k_{n}}\right)$ and $\left(G_{n}^{\prime} F_{k_{n}^{\prime}}^{\prime}\right)$ are homotopic. Hence we can define the composition $[\mathbf{G}] \circ[\mathbf{F}]$ as the homotopy class of the multi-net $\left(G_{n} F_{k_{n}}\right)$. Then it is clear that we get in this way a category whose objects are the compact metric spaces and whose morphisms are the homotopy classes of multi-nets. The identity morphism $1_{X}$ in this category is the homotopy class of $\left(i_{n}\right)$, where $i_{n}=\mathrm{id}_{X}$ for each $n$. We denote the category by $H N$.

We are going to describe a functor $\Omega$ from this category to the shape category of compacta which is an isomorphism of categories. Let $\Omega(X)=X$ for every metric compactum. Suppose $\mathbf{F}=\left(F_{n}\right): X \rightarrow Y$ is a multi-net. We define $\Omega[\mathbf{F}]=\Omega_{X, Y}[\mathbf{F}]$, where $\Omega_{X, Y}$ is the bijection considered in Theorem 2. By virtue of Theorem 2 we only need to prove that $\Omega([\mathbf{G}][\mathbf{F}])=\Omega[\mathbf{G}] \circ \Omega[\mathbf{F}]$. Suppose that $\mathbf{g}=\left\{g_{k}, Y \rightarrow Z\right\}$ is an approximative map which is obtained by an infinitesimal translation from the multi-net $\mathbf{G}=\left(G_{n}\right): Y \rightarrow Z$. By 
Mardešić's Theorem $2^{\prime}$ in [15] there is a fundamental sequence $\hat{\mathbf{g}}=\left\{\hat{g}_{k}, Y, Z\right\}$ such that $\hat{g}_{k \mid Y}=g_{k}$ for every $k$.

Consider a null sequence $\varepsilon_{1} \geq \varepsilon_{2} \geq \cdots \geq \varepsilon_{n} \geq \cdots$ such that

(1) $d\left(g_{n}(y), G_{n}(y)\right)<\varepsilon_{n}$ for every $y \in Y$ and every natural number $n$ and

(2) for every index $n_{0}$ we have that $G_{n}$ is $\varepsilon_{n_{0}}$-multihomotopic to $G_{n_{0}}$ for $n \geq n_{0}$.

Select another null sequence $\mu_{1} \geq \cdots \geq \mu_{n} \geq \cdots$ such that (1) $d\left(\hat{g}_{n}(y), \hat{g}_{n}\left(y^{\prime}\right)\right)$ $<\varepsilon_{n}$ whenever $y$ and $y^{\prime}$ are $\mu_{n}$-close points in $Q$ and $(2) \delta\left(G_{n}(K)\right)<\varepsilon_{n}$ whenever $K$ is a subset of $Y$ with $\delta(K)<\mu_{n}$. Next consider a sequence of indices $k_{1}<k_{2}<\cdots<k_{n}<\cdots$ such that $F_{k}$ and $F_{k_{n}}$ are $\mu_{n}$-multihomotopic for $k \geq k_{n}$. By virtue of the Approximation Lemma there is a sequence of (single-valued) maps $f_{n}: X \rightarrow Q$ such that $d\left(f_{n}, F_{k_{n}}\right)<\mu_{n}$ for every $n \in \mathbf{N}$. We obviously have that $\mathbf{f}=\left\{f_{n}, X \rightarrow Y\right\}$ is an approximative map which is an infinitesimal translation of $\left(F_{k_{n}}\right): X \rightarrow Y$ and $\Omega[\mathbf{F}]=[\mathbf{f}]$.

Moreover, for every point $x \in X$ there is a point $y \in F_{k_{n}}(x)$ such that $d\left(f_{n}(x), y\right)<\mu_{n}$. This implies that $d\left(\hat{g}_{n} f_{n}(x), g_{n}(y)\right)<\varepsilon_{n}$. On the other hand $d\left(g_{n}(y), G_{n} F_{k_{n}}(x)\right) \leq d\left(g_{n}(y), G_{n}(y)\right)<\varepsilon_{n}$. Hence

$$
d\left(\hat{g}_{n} f_{n}(x), G_{n} F_{k_{n}}(x)\right)<2 \varepsilon_{n} .
$$

This shows that $\hat{\mathbf{g}}$ is an approximative map obtained by an infinitesimal translation from the multi-net $\left(G_{n} F_{k_{n}}\right): X \rightarrow Z$ and, as a consequence, $\Omega([\mathbf{G}][\mathbf{F}])=$ $[\hat{\mathbf{g}} \mathbf{f}]=[\mathbf{g}] \circ[\mathbf{f}]=\Omega[\mathbf{G}] \circ \Omega[\mathbf{F}]$. This completes the proof of the theorem.

In our next result we use Theorem 3 to give a necessary and sufficient condition for a map to induce a shape equivalence.

Corollary 5. Let $f: X \rightarrow Y$ be a map of compacta. Then the following statements are equivalent:

(a) $f$ is a shape equivalence.

(b) For every compactum $Z$ and for every $\varepsilon$-small multivalued map $F: X \rightarrow$ $Z$ there is an $\varepsilon$-small multivalued map $G: Y \rightarrow Z$, unique up to $\varepsilon$-multihomotopy, such that $G f$ is $\varepsilon$-multihomotopic to $F$.

Proof. We start by proving the implication (a) $\Rightarrow($ b) . Suppose that $F: X \rightarrow Z$ is an $\varepsilon$-small multivalued map and pick up a $\mu>0$ such that $\delta(F(K))<\varepsilon$ whenever $K$ is a subset of $X$ with $\delta(K)<\mu$. Since $f$ is a shape equivalence, there is by Theorem 3 a multi-net $\mathbf{F}^{\prime}: Y \rightarrow X$ such that $\left[\mathbf{F}^{\prime}\right]$ is the inverse of the homotopy class of the multi-net all of whose terms are equal to $f$. Hence, there is an index $n_{0}$ such that $F_{n_{0}}^{\prime}$ is $\mu$-small and $F_{n_{0}}^{\prime} f$ is $\mu$-multihomotopic to $1_{X}$. This implies that $F F_{n_{0}}^{\prime} f$ is $\varepsilon$-multihomotopic to $F$. Thus, if we define $G=$ $F F_{n_{0}}^{\prime}$ we get an $\varepsilon$-small multivalued map such that $G f$ is $\varepsilon$-multihomotopic to $F$.

Suppose now that $G_{1}$ and $G_{2}$ are $\varepsilon$-small multivalued maps from $Y$ to $Z$ such that $G_{1} f$ and $G_{2} f$ are $\varepsilon$-multihomotopic and consider an $\varepsilon$-small multivalued map $H: X \times I \rightarrow Z$ with $H(x, 0)=G_{1} f(x)$ and $H(x, 1)=$ $G_{2} f(x)$ for every $x \in X$. Select a $\mu>0$ such that

(1) $\delta\left(G_{1}(K)\right)<\varepsilon$ and $\delta\left(G_{2}(K)\right)<\varepsilon$ whenever $K$ is a subset of $Y$ with $\delta(K)<\mu$ and

(2) $\delta(H(K))<\varepsilon$ whenever $K$ is a subset of $X \times I$ with $\delta(K)<\mu$. Consider an index $n_{0}$ such that $F_{n_{0}}^{\prime}$ is $\mu$-small and $f F_{n_{0}}^{\prime}$ is $\mu$-multihomotopic to $1_{Y}$. Then $G_{1} f F_{n_{0}}^{\prime}$ is $\varepsilon$-multihomotopic to $G_{2} f F_{n_{0}}^{\prime}$. On the other hand 
$G_{1} f F_{n_{0}}^{\prime}$ is $\varepsilon$-multihomotopic to $G_{1}$ and $G_{2} f F_{n_{0}}^{\prime}$ is $\varepsilon$-multihomotopic to $G_{2}$. This clearly implies that $G_{1}$ and $G_{2}$ are $\varepsilon$-multihomotopic.

To prove that (b) $\Rightarrow$ (a) suppose that $f^{\prime}: X \rightarrow Z$ is an arbitrary (singlevalued) map with $Z \in$ ANR. Then there is an $\varepsilon>0$ such that every two $\varepsilon$-close maps from a compactum to $Z$ are homotopic. By Corollary 2 there is a $\mu>0(\mu<\varepsilon / 3)$ such that every $\mu$-small multivalued map to $Z$ is $\varepsilon / 3$ close to a single-valued map. Consider a $\mu$-small multivalued map $G: Y \rightarrow Z$ such that $G f$ is $\mu$-multihomotopic to $f^{\prime}$. Then there is a single-valued map $H: X \times I \rightarrow Z$ such that $d\left(H_{0}, G f\right)<\varepsilon / 3$ and $d\left(H_{1}, f^{\prime}\right)<\varepsilon / 3$. There is also a single-valued map $g: Y \rightarrow Z$ such that $d(g, G)<\varepsilon / 3$. Hence $d\left(g f, H_{0}\right)<\varepsilon$ and $g f \simeq H_{0} \simeq H_{1} \simeq f^{\prime}$.

Suppose now that $g_{1}, g_{2}: Y \rightarrow Z$ are maps such that $g_{1} f \simeq g_{2} f$. Then $g_{1}$ is $\varepsilon$-multihomotopic to $g_{2}$ for every $\varepsilon$ and by Corollary $3, g_{1} \simeq g_{2}$. Then according to [7, Theorem 4.3.1], $f$ is a shape equivalence. This completes the proof of the corollary.

We shall now show how to use our Corollary 5 to give a simple proof of the main result of [10], due to Kato.

Corollary 6 (Kato). If $r: X \rightarrow Y$ is a refinable map and $Y \in$ FANR then $r$ is a shape equivalence.

Proof. Suppose $F: X \rightarrow Z$ is an $\varepsilon$-small multivalued map and select a $\mu>0$ such that $\delta(F(K))<\varepsilon$ whenever $K$ is a subset of $X$ with $\delta(K)<\mu$. Since $Y \in$ FANR there is by Corollary 4 a $\mu^{\prime}<\mu$ such that if $r^{\prime}$ is a $\mu^{\prime}$-refinement of $r$, then $r$ and $r^{\prime}$ are $\eta$-multihomotopic for every $\eta>0$. Let $F^{\prime}=\left(r^{\prime}\right)^{-1}$ and define $G=F F^{\prime}$. Obviously $G$ is an $\varepsilon$-small multivalued map and $G r=F F^{\prime} r$ is $\varepsilon$-multihomotopic to $F F^{\prime} r^{\prime}$. But $F^{\prime} r^{\prime}$ is $\mu^{\prime}$-multihomotopic to $1_{X}$ and, as a consequence, $G r$ is $\varepsilon$-multihomotopic to $F$.

Suppose now that $G_{1}$ and $G_{2}$ are $\varepsilon$-small multivalued maps from $Y$ to $Z$ such that $G_{1} r$ and $G_{2} r$ are $\varepsilon$-multihomotopic. Then there is a $\mu>0$ such that $\delta\left(G_{1}(K)\right)<\varepsilon$ and $\delta\left(G_{2}(K)\right)<\varepsilon$ whenever $K$ is a subset of $Y$ with $\delta(K)<\mu$. By Corollary 1 there is a $\mu^{\prime}>0$ such that every two $\mu^{\prime}$-close and $\mu^{\prime}$-small multivalued maps to $Y$ are $\mu$-multihomotopic. Since $r$ is refinable we can find a $\mu^{\prime}$-refinement $r^{\prime}$ of $r$ such that if $F=\left(r^{\prime}\right)^{-1}: Y \rightarrow X$ then $r F$ is $\mu^{\prime}$-small and $\mu^{\prime}$-close to $1_{Y}$. Moreover it is possible to achieve that $F$ be sufficiently small so that $G_{1} r F$ and $G_{2} r F$ are $\varepsilon$-multihomotopic. Obviously this implies that $G_{1}$ is $\varepsilon$-multihomotopic to $G_{2}$ and according to Corollary 5, $r$ is a shape equivalence. This completes the proof of the corollary.

We close this section by giving a characterization of the inclusions inducing strong shape equivalences. We express our result in terms of multivalued analogues of strong deformation retractions. We use in the proof Dydak and Segal characterization of the inclusions which induce strong shape equivalences (see $[8$, Theorem 6.2]).

Theorem 4. Let $X \subset Y$ be compacta. Then the following conditions are equivalent:

(a) the inclusion map $i: X \rightarrow Y$ induces a strong shape equivalence.

(b) for every $\varepsilon>0$ there is an $\varepsilon$-small multivalued map $F: Y \times I \rightarrow Y$ such that $y \in F(y, 0)$ and $F(y, 1) \subset X$ for all $y \in Y$ and $x \in F(x, t)$ for all $x \in X$ and $0 \leq t \leq 1$. 
Proof. Let $V=B(Y, \varepsilon / 3)$ and $U=B(X, \varepsilon / 3)$, where the balls are taken in the Hilbert cube, $Q$. By [8, Theorem 6.2], there is a homotopy $H: Y \times I \rightarrow V$ such that $H(y, 0)=y$ and $H(y, 1) \in U$ for every $y \in Y$ and $H(x, t)=x$ for every $x \in X$ and $0 \leq t \leq 1$. Define $F^{\prime}: Y \times I \rightarrow Y$ by the expression $F^{\prime}(y, t)=\{z \in Y \mid d(z, \bar{H}(y, t)) \leq \varepsilon / 3\}$. Then $F^{\prime}$ is an $\varepsilon$-small multivalued map such that $y \in F^{\prime}(y, 0)$ for every $y \in Y$ and $x \in F^{\prime}(x, t)$ for every $x \in X$ and $0 \leq t \leq 1$. Consider the multivalued map $G: Y \rightarrow X$ defined by $G(y)=F^{\prime}(y, 1) \cap \bar{X}$. Then we can define an $\varepsilon$-multihomotopy $F^{\prime \prime}: Y \times I \rightarrow Y$ by the expressions $F^{\prime \prime}(y, 0)=F^{\prime}(y, 1)$ and $F^{\prime \prime}(y, t)=G(y)$ for every $y \in Y$ and $0<t \leq 1$. Now by combining the homotopies $F^{\prime}$ and $F^{\prime \prime}$ it is easy to get a multivalued map $F: Y \times I \rightarrow Y$ fulfilling the required conditions.

To prove the converse take ANR-neighborhoods $V$ of $Y$ in $Q$ and $U \subset V$ of $X$ in $Q$. Consider an $\varepsilon>0$ such that $B(Y, \varepsilon) \subset V, B(X, \varepsilon) \subset U$ and every two $\varepsilon$-close mappings from a compactum to $V$ are homotopic. By [2, Theorem 3.1], there is a $\mu>0,(\mu<\varepsilon)$, such that if $f$ and $g$ are $\mu$-close maps from $X$ to $U$ and $f$ can be extended to a map $\hat{f}: Y \rightarrow U$, then $g$ can also be extended to a map $\hat{g}: Y \rightarrow U$ with $d(\hat{f}, \hat{g})<\varepsilon$. Consider a $\mu / 2$-small multivalued map $F: Y \times I \rightarrow Y$ such that $y \in F(y, 0)$ and $F(y, 1) \subset X$ for every $y \in Y$ and $x \in F(x, t)$ for every $x \in X$ and $0 \leq t \leq 1$. By the Approximation Lemma there is a (single-valued) map $H: Y \times I \rightarrow Q$ such that $d(H, F)<\mu / 2$. Hence $H$ takes values in $V$ and $H(y, 1) \in U$ for every $y \in Y$. Since $d(H(x, 1), x)<\mu$ for every $x \in X$ there is a map $g: Y \rightarrow U$ such that $g_{\mid X}=1_{X}$ and $d(g(y), H(y, 1))<\varepsilon$ for every $y \in Y$. Hence $H_{\mid Y \times\{0\} \cup X \times I \cup Y \times\{1\}}$ is $\varepsilon$-close to the map $G$ such that $G(y, 0)=y$ and $G(y, 1)=g(y)$ for every $y \in Y$ and $G(x, t)=x$ for all $x \in X$ and $0 \leq t \leq 1$, hence they are homotopic in $V$ and by the homotopy extension theorem there exists a map $\widehat{G}: Y \times I \rightarrow V$ such that $\widehat{G}_{\mid Y \times\{0\} \cup X \times I \cup Y \times\{1\}}=G$. Then, by virtue of [8, Theorem 6.2], $i$ is a strong shape equivalence. This completes the proof of the theorem.

\section{Multi-NetS AND INTERNALly MOVABLE COMPACTA}

Internally movable compacta were introduced by Bogatyi in [1]. A compactum $X$ lying in the Hilbert cube, $Q$, is said to be internally movable provided that for every neighborhood $U$ of $X$ in $Q$ there is another neighborhood $U_{0} \subset U$ that can be deformed into $X$ in $U$. Dydak proved in [6] that every movable shape admits in internally movable representative. Other properties of internally movable compacta in connection with shape morphisms were established in [12 and 18].

In this section we study in detail the homotopy classes of multi-nets between internally movable compacta and prove that they admit an especially nice kind of representatives that we call simple multi-nets. In order to prove this result we must first give a new characterization of internally movable compacta in terms of multivalued maps. In a forthcoming paper we shall give similar characterizations of the most usual concepts of the theory of shape and also of the notions related to the limits of shape maps considered in [19].

Theorem 5. A metric compactum $X$ is internally movable if and only if for every $\varepsilon>0$ there is a $\mu>0$ such that every $\mu$-small multivalued map $F: Y \rightarrow X$ 
(where $Y$ is an arbitrary compactum) is $\varepsilon$-multihomotopic to a single-valued map.

Proof. Let $\varepsilon>0$ be given and suppose that $X$ is internally movable. Consider a neighborhood $U$ of $X$ in $Q$ such that $U \subset B(X, \varepsilon / 3)$ and let $U_{0}$ be the neighborhood that realizes in $U$ the internal movability of $X$. Select a $\delta>0,(\delta<\varepsilon / 6)$, such that $B(X, \delta) \subset U_{0}$. Suppose $F: Y \rightarrow X$ is a $\delta$-small multivalued map. By the Approximation Lemma there is a single-valued map $f: Y \rightarrow Q$ such that $d(f, F)<\delta$. Obviously $f(Y) \subset U_{0}$. Consider a (singlevalued) map $H: Y \times I \rightarrow U$ such that $H(y, 0)=f(y)$ and $H(y, 1) \in X$ for every $y \in Y$. Now define a multivalued map $\widehat{H}: Y \times I \rightarrow X$ by $\widehat{H}(y, t)=$ $\{x \in X \mid d(x, H(y, t)) \leq \varepsilon / 3\}$. Obviously $\widehat{H}$ is $\varepsilon$-small and $F(y) \subset \widehat{H}(y, 0)$ and $H(y, 1) \subset \widehat{H}(y, 1)$ for every $y \in Y$. Then it is easy to see that $F$ is $\varepsilon$-multihomotopic to the single-valued map $h: Y \rightarrow X$ defined by $h(y)=$ $H(y, 1)$.

Conversely, suppose that for every $\varepsilon>0$ there is a $\delta>0, \delta<\varepsilon$, such that every $\delta$-small multivalued map $F: Y \rightarrow X$ is $\varepsilon$-multihomotopic to a single-valued map. Let $U$ be an ANR-neighborhood of $X$ in $Q$ and select an $\varepsilon>0$ such that (1) $B(X, \varepsilon) \subset U$ and (2) every two $2 \varepsilon$-close maps into $U$ are homotopic. Let $U_{0}$ be a compact neighborhood of $X$ in $Q$ such that $U_{0} \subset B(X, \delta / 3)$ and define a multivalued map $F: U_{0} \rightarrow X$ by $F(u)=\{x \in$ $X \mid d(x, u) \leq \delta / 3\}$. Then $F$ is $\delta$-small and there is an $\varepsilon$-multihomotopy $H: U_{0} \times I \rightarrow X$ such that $H(u, 0)=F(u)$ for every $u \in U_{0}$ and $H_{1}$ is single-valued. By the Approximation Lemma there is a (single-valued) map $G: U_{0} \times I \rightarrow Q$ such that $d(G(u, t), H(u, t))<\varepsilon$ for every $u \in U_{0}$ and every $0 \leq t \leq 1$. Obviously $G\left(U_{0} \times I\right) \subset U$. Moreover $d(G(u, 0), u)<2 \varepsilon$. This clearly implies that the inclusion $j: U_{0} \rightarrow U$ is homotopic in $U$ to the map $H_{1}: U_{0} \rightarrow X$ and, as a consequence, $X$ is internally movable.

Definition 3. A multi-net $\mathbf{F}=\left(F_{n}\right): X \rightarrow Y$ is said to be simple if $F_{n}$ is singlevalued for every $n$.

Remark. The fact that $\mathbf{F}=\left(F_{n}\right): X \rightarrow Y$ is a simple multi-net does not in general imply that the $\varepsilon$-multihomotopies connecting its terms are single-valued.

With the help of Theorem 5 we shall be able to prove our following result which allows us to give a nice representation of the shape morphisms between internally movable compacta.

Theorem 6. Let $X$ and $Y$ be metric compacta. If $Y$ is internally movable, then every homotopy class of multi-nets between $X$ and $Y$ has a simple representative.

Proof. Let $\mathbf{F}=\left(F_{n}\right): X \rightarrow Y$ be a multi-net and consider a null sequence $\varepsilon_{1} \geq \cdots \geq \varepsilon_{n} \geq \cdots$ of positive numbers. Since $Y$ is internally movable there is a null sequence $\delta_{1} \geq \cdots \geq \delta_{n} \geq \cdots$ such that every $\delta_{n}$-small multivalued map into $Y$ is $\varepsilon_{n}$-multihomotopic to a single-valued map. Consider a sequence of indices $k_{1}<\cdots<k_{n}<\cdots$ such that $F_{k}$ is $\delta_{n}$-multihomotopic to $F_{k_{n}}$ for every $k \geq k_{n}$. Then, we can define a sequence of single-valued maps $F_{n}^{\prime}: X \rightarrow Y$ such that $F_{k}^{\prime}$ is $\varepsilon_{n}$-multihomotopic to $F_{k}$ whenever $k_{n} \leq k<k_{n+1}$. It can be easily proved that $\mathbf{F}^{\prime}=\left(F_{n}^{\prime}\right): X \rightarrow Y$ is a simple multi-net homotopic to $\mathbf{F}$. This completes the proof of the theorem. 
Example 1. Consider in the Euclidean plane $E^{2}$ the circles $X_{n}=\left\{(x, y) \mid x^{2}+\right.$ $\left.y^{2}=(1-1 / n+1)^{2}\right\}$ for $n \geq 1$ and let $X_{0}$ be the circle with centre $(0,0)$ and radius 1 . We set $X=\bigcup_{n=0}^{\infty} X_{n}$. Now, consider the points $p_{1}=(0,0)$, $p_{2 m}=(1-1 / m+1,0), p_{2 m+1}=((1 / m+1)-1,0)$ for each $m \geq 1$, and let $\left(Y_{n}\right)_{n \geq 0}$ be a sequence of mutually disjoint circles such that $Y_{0}=X_{0}$, the centre of $Y_{n}$ is $p_{n}($ for $n \geq 1)$ and $\lim \cdot \operatorname{radius}\left(Y_{n}\right)=0$. We set $Y=\bigcup_{n=0}^{\infty} Y_{n}$. It is easy to see that $X$ and $Y$ are internally movable compacta.

Consider a sequence of maps $F_{n}: X \rightarrow Y$ such that:

(1) for every $n \geq 1, F_{n}$ maps homeomorphically $X_{i}$ onto $Y_{i}$, if $1 \leq i \leq n$, and maps $X_{0}$ and $X_{i}$ to the point $p=(1,0)$, if $i>n$.

(2) $F_{n \mid X_{i}}=F_{m \mid X_{i}}$ if $i \leq \min \{n, m\}$.

It is easy to see that $\mathbf{F}=\left(F_{n}\right): X \rightarrow Y$ is a simple multi-net that represents the shape morphism corresponding to the homotopy class of the approximative map $\mathbf{f}=\left\{f_{k}, X \rightarrow Y\right\}$, where $f_{k}=F_{k}$ for every $k$. We now prove that this shape morphism is not generated by a map.

Otherwise, if $\mathbf{f}$ were homotopic to an approximative map, $\mathbf{g}$, generated by a map $g: X \rightarrow Y$, then, the induced maps, $\Lambda_{\mathrm{f}}, \Lambda_{\mathrm{g}}$, between the spaces of components $\square(X)$ and $\square(Y)$ would be coincident (see Borsuk [4, p. 214]), and we should have $\Lambda_{\mathrm{g}}\left(X_{n}\right)=\Lambda_{\mathrm{f}}\left(X_{n}\right)=Y_{n}$ and, therefore $g\left(X_{n}\right) \subset Y_{n}$ for every $n \geq 0$. Let $x_{n} \in X_{n}$ be the point $(1-1 / n+1,0)$ for each index $n \geq 1$; then, the sequences $\left(x_{2 n}\right)$ and $\left(x_{2 n+1}\right)$ converge to the point $p$ but $\lim . g\left(x_{2 n}\right)=p$, $\lim . g\left(x_{2 n+1}\right)=p^{\prime}=(-1,0)$. This contradicts the fact that $g$ is continuous.

Finally, we present two results giving characterizations of quasi-equivalence and shape equivalence for internally movable compacta and internal FANR's respectively. The notion of quasi-equivalence was introduced by Borsuk in [2]. Two compacta $X$ and $Y$ are quasi-equivalent if for every pair of maps $h: X \rightarrow M, l: Y \rightarrow N$, where $M, N \in$ ANR, there are shape morphisms $\mu: X \rightarrow Y, \eta: Y \rightarrow X$ such that $S(h) \circ \eta \circ \mu=S(h), S(l) \circ \mu \circ \eta=S(l)$, where $S(h)$ and $S(l)$ are the shape morphisms induced by $h$ and $l$ respectively. Internal FANR's were introduced in [12] and they agree with compacta which are simultaneously FANR's and internally movable. The proofs of the next results will be left to the reader and depend on Theorem 6 and on the fact, proved by Dydak in [6], that every shape morphism from a compactum to an internal FANR is generated by a map.

Theorem 7. Two internally movable compacta $X$ and $Y$ are quasi-equivalent if and only if for every $\varepsilon>0$ there are single-valued maps $f: X \rightarrow Y$ and $g$ : $Y \rightarrow X$ such that $g f$ is $\varepsilon$-multihomotopic to $1_{X}$ and $f g$ is $\varepsilon$-multihomotopic to $1_{Y}$.

Theorem 8. Two internal FANR's $X$ and $Y$ are shape equivalent if and only if there are continuous maps $f: X \rightarrow Y$ and $g: Y \rightarrow X$ such that $g f$ is $\varepsilon$-multihomotopic to $1_{X}$ and $f g$ is $\varepsilon$-multihomotopic to $1_{Y}$ for every $\varepsilon>0$.

\section{REFERENCES}

1. S. Bogatyi, Approximate and fundamental retracts, Math. Sb. 22 (1974), 91-103.

2. K. Borsuk, Theory of retracts, Monogr. Mat. 44, Polish Scientific Publishers, Warszawa, 1967.

3. _ Concerning homotopy properties of compacta, Fund. Math. 62 (1968), 223-254. 
4. __ Theory of shape, Monogr. Mat. 59, Polish Scientific Publishers, Warszawa, 1975.

5. __ Some quantitative properties of shapes, Fund. Math. 93 (1976), 197-212.

6. J. Dydak, On internally movable compacta, Bull. Acad. Polon. Sci. 27 (1979), 107-110.

7. J. Dydak and J. Segal, Shape theory: An introduction, Lecture Notes in Math., vol. 688, Springer-Verlag, Berlin, 1978.

8. __ Strong shape theory, Dissertationes Math. 192 (1981), 1-42.

9. J. E. Felt, E-continuity and shape, Proc. Amer. Math. Soc. 46 (1974), 426-430.

10. H. Kato, Refinable maps in the theory of shape, Fund. Math. 113 (1981), 119-129.

11. V. L. Klee and A. Yandl, Some proximate concepts in topology, Symposia Math., vol. 16 (INDAM, Rome, 1973), Academic Press, London, 1974, pp. 21-39.

12. V. F. Laguna and J. M. R. Sanjurjo, Internal fundamental sequences and approximative retracts, Topology Appl. 17 (1984), 189-197.

13. __ Spaces of approximative maps, Math. Japon. 31 (1986), 623-633.

14. __ Shape morphisms and spaces of approximative maps, Fund. Math. 183 (1989), 225235.

15. S. Mardešić, On Borsuk's shape theory for compact pairs, Bull. Acad. Polon. Sci. 21 (1973), $1131-1136$.

16. S. Mardešić and J. Segal, Shapes of compacta and ANR-systems, Fund. Math. 72 (1971), 41-59.

17. __ Shape theory, North-Holland, Amsterdam, 1982.

18. M. A. Moron, On internal movability, internal shape and internal MANR-spaces, Colloq. Math. (to appear).

19. J. M. R. Sanjurjo, On limits of shape maps, Topology Appl. 23 (1986), 173-181.

20. __ A non-continuous description of shape, Quart. J. Math. Oxford Ser. 40 (1989), 351359.

21. __ Shape morphisms and small multivalued maps, preprint.

Departamento de Geometria y Topologia, Facultad de Matematicas, Universidad Complutense, 28040 Madrid, SPain 